\title{
Fast implementation of root-form eigen-based methods for detecting closely spaced sources
}

\author{
Ta-Sung Lee
}

Indexing terms: Algorithms, Signal processing

\begin{abstract}
Root-form eigen-based methods for direction-of-arrival (DOA) estimation represent a class of estimators that exhibit a higher resolution capability relative to spectral-form estimators in detecting closely spaced sources with a linear, equally spaced (LES) array. These methods require an eigenvalue decomposition (EVD) and a polynomial rooting. Although the numerical complexity associated with the EVD is greatly reduced with the use of beamspace transformation, largeorder polynomial rooting still raises practical difficulties. As a remedy, the author proposes a novel iterative implementation of beamspace root-form methods without the need for large-order polynomial rooting. The new method exploits the banded structure of the augmented noise eigenvector matrix associated with an LES array. It requires only rooting in parallel several smallorder polynomials and some minor matrix manipulations at each iteration. It is shown that the proposed method offers the performance of beamspace root-MUSIC.
\end{abstract}

\section{Introduction}

Eigen-based methods for DOA estimation represent a class of techniques that offer a much better resolution performance than that of conventional beamformers. In eigen-based methods, signal and noise subspaces are identified first via an $M \times M$ generalised EVD (GEVD) of the array data/noise correlation matrix pencil, where $M$ equals the number of array elements. A search is then conducted over a null spectrum associated with the noise subspace, to locate the minima, from which the source DOAs can be determined. In the case where an LES array is employed, the null-spectrum searching can be converted into a polynomial rooting problem. 'Two well known examples are the root-MUSIC [1] and rootminimum-norm [2] methods. They belong to the socalled weighted root-form eigen-based methods. Compared to their spectrum-searching or spectral-form counterparts, root-form methods exhibit a higher resolution capability in dealing with closely spaced sources. Rao and Hari [3] argue that a zero of the null spectrum, having a large radial error, will cause the corresponding spectral minima to be less defined, but does

Paper 8875 F (E5), first received 21st August 1991 and in revised form 24th March 1992

The author is with the Department of Communication Engineering,

National Chiao Tung University, Hsinchu, Taiwan, Republic of China not affect the DOA estimates. As for the mean-squared errors of the DOA estimates, $\mathrm{Li}$ and Vaccaro [4] show that both spectral and root-form methods yield the same expression. It should be borne in mind, however, that the result holds only when each of the sources has a minimum corresponding to it in the null spectrum.

A major issue regarding eigen-based methods is the heavy computational load associated with the GEVD. This is more significant when $M$ is large. To remedy this, the concept of beamspace transformation was proposed [5] as a means of reducing the dimension of the array data. In beamspace processing, the $M \times 1$ 'element-space' data vectors are transformed into $K \times 1$ 'beamspace' data vectors via an $M \times K$ matrix beamformer, where $K$ is assumed to be greater than the number of sources $D$. Eigen-based methods applied to these reduced-dimension beamspace data then require only a GEVD of the $K \times K$ beamspace data/noise correlation matrix pencil, leading to a substantial reduction in computation when $K$ is much less than $M$. In addition, processing in the beamspace can also improve the resolution-SNR (signal-tonoise ratio) threshold, enhance the estimation performance and reduce sensitivity to the spatial noise correlation and errors in the array manifold. Lee and Wengrovitz [6] show that, by judiciously choosing a $M \times 3$ beamformer, the resolution-SNR threshold of the MUSIC method dealing with two closely spaced sources can be reduced by a factor of $M-2$. In the light of these advantages and the efficacy of root-form methods, a beamspace root-form eigen-based scheme is highly desirable.

A practical case of importance that receives little attention in the area of array processing is the development of a high-performance and yet computationally efficient algorithm for the localisation of a group of closely spaced sources. We find, from the above observations, that the beamspace root-form eigen-based method is a good candidate for this purpose, provided that an LES array is available. Unfortunately, such methods usually require a $2(M-1)$ th-order (for root-MUSIC) or an $(M-1)$ th-order (for root-minimum-norm) polynomial rooting as the final stage in determining the DOAs. This becomes inefficient as $M$ becomes much greater than $D$. In practice, phased arrays may have up to 100 elements and may be operated to track a group of few targets. This makes the direct application of beamspace root-MUSIC or root-minimum-norm too costly. Research efforts have been made to remedy the difficulty of large-order polynomial rooting $[7,8]$. In Reference 8 , the common out-of-band nulls property associated with a set of Butler-type beams [9] is exploited as a means of reducing the $2(M-1)$ th-order root-MUSIC polynomial into a $2(K-1)$ th-order one. There are, however, two reasons 
for not restricting ourselves to the use of the Butler beamformer. First, it is costly to implement on a system with large $M$. Secondly the Butler beamformer does not achieve the best performance in resolving tightly spaced sources. A scheme that employs a specifically constructed beamformer and does not require rooting a large-order polynomial is thus required. In this paper, we propose a novel implementation of beamspace root-form eigenbased methods. The new method exploits the distinctive banded structure of the augmented noise eigenvector (EV) matrix and the Vandermonde structure of the array manifold vector associated with an LES array. The noise EV matrix has a bandwidth of $D+1$, such that the resulting null spectrum can be manipulated into a reduced form corresponding to a $(D+1)$-element LES array. As a consequence, the final DOA estimates can be determined by rooting either a $2 D$ th- or $D$ th-order polynomial. Since the construction of the augmented noise EV matrix and the subsequent polynomial rooting require the knowledge of the DOAs, an iterative implementation is suggested. To further improve the estimation accuracy, we suggest a scheme that determines each of the DOAs individually, by rooting in parallel $D 2 D$ thor $D$ th-order polynomials, i.e. a $2 D$ th-or $D$ th-order polynomial rooting is executed to determine a single DOA estimate. The new method offers the performance of beamspace root-MUSIC, but requires a much lower computational load. The algorithm converges within a few iterations and is not sensitive to the errors in the initial DOA estimates. The most computationally demanding part of the algorithm involves concurrently solving $K-D$ systems of linear equations of size $M-D$ Fortunately, these systems of equations are sparse, and banded in structure such that fast solution can be easily achieved.

\section{Beamspace root-form eigen-based methods}

We here consider the scenario of $D$ narrowband sources with a common centre frequency $f_{c}$ impinging on an LES array of $M$ identical sensor elements. The sources are assumed to be in the far field of the array, such that the planewave assumption holds at each element. We further assume that the sensor elements have a common passband centred at $f_{c}$. Additive noise uncorrelated with the source field, with a known cross-spectral density, is also assumed to be present at each element. The complex envelopes of the array data received at the $M$ elements can be expressed in the following vector form

$$
x(t)=A s(t)+n(t)
$$

The $m$ th component of the $M \times 1$ array data vector $x(t)$ represents the complex envelope received at the $m$ th element. The $i$ th component of the $D \times 1$ source signal vector $s(t)$ represents the complex envelope received at some reference point of the array due to the $i$ th source The ith column of the $M \times D$ DOA matrix $A$ accounts for the phase variation across the entire array due to the planewave nature of the $i$ th source. Finally, the $m$ th component of the $M \times 1$ vector $n(t)$ denotes the complex noise envelope present at the $m$ th element. The $i$ th column of $A$ has a distinctive Vandermonde structure described by

$$
a(u)=\left[1, e^{j \pi\left(f_{c} / f_{0}\right) u}, e^{j 2 \pi\left(f_{c} / f_{o}\right) u}, \ldots, e^{j(M-1) \pi\left(f_{\mathrm{c}} / f_{0}\right) u}\right]^{T}
$$

with $u=u_{i}=\sin \left(\theta_{i}\right)$, where $\theta_{i}$ is the DOA of the $i$ th source measured with respect to the broadside of the array. $f_{o}$ is the frequency corresponding to halfwavelength array element spacings. For convenience, we will assume $f_{c}=f_{o}$. Note that we have set the reference point of the array to be at its first element.

In beamspace array signal processing, the data envelopes are obtained from a set of $K$ beamformer ports. This may be accomplished by using a set of $K$ beamforming weight vectors to simultaneously form $K$ linear combinations of the received array data. Mathematically speaking, we transform the $M \times 1$ element space array data vector into a $K \times 1$ beamspace data vector according to

$$
\boldsymbol{x}_{B}(t)=W^{H} \boldsymbol{x}(t)
$$

where $W$ is the $M \times K$ beamforming matrix having the form

$$
W=\left[w_{1}\left|w_{2}\right| \cdots \mid w_{K}\right]
$$

where $w_{k}$ is the $k$ th beamforming weight vector. Here, $K$ is chosen to be such that $D<K \leqslant M$. Note that $x_{B}(t)$ has the same structural form as $\boldsymbol{x}(t)$

$$
x_{B}(t)=W^{H}[A s(t)+n(t)]=B s(t)+n_{B}(t)
$$

where $B=W^{H} A$ is the beamspace DOA matrix whose $i$ th column is the beamspace manifold vector $b(u)=W^{H} a(u)$ evaluated at $u=u_{i}$, and $n_{B}(t)=W^{H} m(t)$ is the beamspace noise vector. Note that $b(u)$ does not possess a Vandermonde structure. To facilitate discretetime processing, $\boldsymbol{x}_{B}(t)$ is sampled over a time interval to produce a set of $N$ beamspace data 'snapshot' vectors $\boldsymbol{x}_{B}[n]=\boldsymbol{x}_{B}\left(n T_{s}\right), n=1, \ldots, N$, where $T_{s}$ is the sampling period. The general beamspace DOA estimation problem is then formulated as one that estimates the DOAs $u_{i}$, $i=1, \ldots, D$, based on the $N$ vector observations $x_{B}[n]$, $n=1, \ldots, N$.

A class of DOA estimators that exhibit high resolution capability and excellent asymptotical performance are those based on the principle of eigen decomposition. A common feature of these eigen-based methods is the execution of the GEVD of the data/noise correlation matrix pencil. For the beamspace case, the data correlation matrix has the following structural form

$$
\boldsymbol{R}_{B B}=E\left\{x_{B}(t) x_{B}^{H}(t)\right\}=B R_{s s} B^{H}+Q_{B B}
$$

where $R_{\mathrm{ss}}=E\left\{s(t) s^{H}(t)\right\}$ is the $D \times D$ source correlation matrix, $Q_{B B}=E\left\{n_{B}(t) n_{B}^{H}(t)\right\}$ is the $K \times K$ beamspace noise correlation matrix, and $H$ denotes the complex conjugate transpose. In practice, $\boldsymbol{R}_{B B}$ is not available and is typically estimated by its sample version

$$
\hat{\boldsymbol{R}}_{B B}=\frac{1}{N} \sum_{n=1}^{N} \boldsymbol{x}_{B}[n] \boldsymbol{x}_{B}^{H}[n]
$$

Beamspace eigen-based procedures usually consist of the following steps. First, the GEVD of the $K \times K$ pencil $\left\{\widehat{\boldsymbol{K}}_{B B}, \boldsymbol{Q}_{B B}\right\}$ is computed. Secondly, a set of noise EVs is determined as those generalised eigenvectors (GEV) associated with the $K-D$ smallest GEVs. Let $\bar{E}_{B}$ denote the $K \times(K-D)$ matrix composed of these noise EVs. Thirdly, the noise EVs are ideally orthogonal to each of the $D$ columns of $\boldsymbol{B}$, such that $\vec{E}_{B}^{H} \boldsymbol{b}\left(u_{i}\right)=\mathbf{0}, i=1, \ldots, D$. This suggests that we determine the DOAs by solving the following nonlinear equation in $u$

$$
S_{B}(u)=b^{H}(u) \bar{E}_{B} P \bar{E}_{B}^{H} b(u)=0
$$

where $\boldsymbol{P}$ is a positive semidefinite matrix serving to weight the respective noise EVs. Converting back into 
element space terms, we have

$$
\begin{aligned}
S_{B}(u) & =a^{H}(u) W \bar{E}_{B} P \bar{E}_{B}^{H} W^{H} a(u) \\
& =a^{H}(u) \bar{E}_{W} P \bar{E}_{W}^{H} a(u)=0
\end{aligned}
$$

where $\bar{E}_{W}=W \bar{E}_{B}$. Note that we have used $b(u)=W^{H} a(u)$. The expression in eqn. 9 is referred to as the weighted beamspace null spectrum. As two popular methods, beamspace MUSIC corresponds to $P=I$ and beamspace minimum-norm corresponds to $\boldsymbol{P}=\boldsymbol{c} c^{H}$, where $c$ is the transpose of the first row of $\bar{E}_{W}$. Eqn. 9 may not hold for any $u$ in practice, due to noise/error corruption, which may arise from electronic thermal motions, external interferences, array shape perturbations, and finite snapshot formulations of the data correlation matrix, etc. A direct implementation of beamspace MUSIC or minimum-norm, then, is to search over $u$ to locate the minima of $S_{B}(u)$. This is referred to as the spectral-form realisation. As an alternative, we may convert $S_{B}(u)$ into a polynomial of the order $2(M-1)$, by exploiting the Vandermonde structure of $a(u)$. Letting $v(z)=\left[1, z, \ldots, z^{M-1}\right]^{T}$, we can rewrite eqn. 9 as

$$
S_{B}(z)=v^{T}\left(z^{-1}\right) \bar{E}_{W} P \bar{E}_{W}^{H} v(z)=0
$$

with $z=e^{j \pi u}$. For brevity, we refer to $S_{B}(z)$ as the "signal polynomial'. An obvious way to obtain the DOA estimates is to root $S_{B}(z)$ and pick $D$ 'signal roots' $\hat{z}_{i}, i=1, \ldots$, $D$, which are closest to the unit circle. The DOA estimates are then related to the signal roots by $\hat{u}_{i}=$ $(1 / \pi) \arg \left\{\hat{z}_{i}\right\}$. This is referred to as the root-form realis ation. Note again that, due to noise/error corruption, the signal roots may not lie exactly on the unit circle. The comparative study of spectral and root-form methods [3] shows that the latter exhibit a lower resolution-SNR threshold in resolving two closely spaced sources. For well separated sources, the two methods are statistically identical, i.e. the mean-squared errors of the DOA estimates obtained with the two methods are the same [4].

Although beamspace transformation greatly reduces the eigenanalysis dimension, to determine the DOAs via the root-form approach requires rooting a $2(M-1)$ thorder signal polynomial. As a practical matter, the number of elements $M$ may be so large that rooting a polynomial of the order $2(M-1)$ becomes infeasible in real-time applications. Moreover, only $D$ of the $2(M-1)$ roots are needed, such that directly rooting the signal polynomial seems to be very inefficient. Research efforts have been made to develop algorithms that do not require rooting the signal polynomial $[7,8]$. In Reference 8 , the property of common nulls of the Butler type beamformer was utilised to convert the beamspace rootMUSIC signal polynomial into a smaller one of the order $2(K-1)$. The Butler beamformer is an analogue equivalent of the fast Fourier transform which simultaneously forms $M$ beams pointed at equally spaced angles. It requires $(M / 2) \log _{2} M$ hybrids and $(M / 2)\left(\log _{2} M-1\right)$ fixed phase shifters [9]. For the beamforming network to operate losslessly, $M$ must be a power of two. Owing to its hardware complexity, the Butler network is not quite suitable for large arrays. Another issue regarding the Butler beamformer is the high sidelobes due to uniform weighting. This can be alleviated by combining the beams with additional circuitry to obtain an effective lower sidelobe weighting. Unfortunately, the modified network is no longer lossless. Finally, from the viewpoint of beamspace DOA estimation, the Butler beamformer does not offer the best resolution performance [6]. The flexibility of forming a set of 'optimum' beams is obviously not pos- sessed by the Butler beamformer. A computationally efficient beamspace root-form scheme that works in conjunction with any specifically constructed beamformer is thus desirable.

\section{Simplifications due to polynomial order reduction}

Simplifications in computation due to polynomial order reduction is motivated by the characteristic structure of $\operatorname{Null}\left\{A^{H}\right\}$, or the noise subspace, where $\operatorname{Null}\{\cdot\}$ denotes null space. Recall that $A$ is an $M \times D$ full-rank Vandermonde matrix. A basis of $\operatorname{Null}\left\{A^{H}\right\}$ may be chosen as composed of the columns of the following $M \times(M-D)$ banded Toeplitz matrix

$$
\boldsymbol{G}=\left[\begin{array}{cccc}
\boldsymbol{g} & \mathbf{0} & \cdots & 0 \\
0 & \boldsymbol{g} & \cdots & 0 \\
\vdots & \vdots & \ddots & \vdots \\
0 & 0 & \cdots & \boldsymbol{g}
\end{array}\right]
$$

where $g=\left[1, \gamma_{1}, \ldots, \gamma_{D}\right]^{T}$ is a $(D+1) \times 1$ vector satisfying

$$
\sum_{m=0}^{D} \gamma_{m}^{*} e^{j m x u_{i}}=0 \quad i=1, \ldots, D
$$

with $\gamma_{0}=1$, and 0 is a scalar. This is a manifestation of the fact that the polynomial

$$
g(z)=\sum_{m=0}^{D} \gamma_{m}^{*} z^{m}
$$

has $D$ roots at $z_{i}=e^{j \pi u_{i}}, i=1, \ldots, D$. A one-to-one relationship between $\operatorname{Null}\left\{A^{H}\right\}, g, g(z)$ and the DOAs can thus be established. The feasibility of working with $\operatorname{Null}\left\{A^{H}\right\}$ to determine the DOAs, of course, lies in the fact that $\operatorname{Null}\left\{A^{H}\right\}$ is properly estimated.

\subsection{Element space implementation}

In conventional element space eigen-based methods, $\operatorname{Null}\left\{A^{H}\right\}$ is estimated as spanned by the GEVs of the data/noise correlation matrix pencil, associated with the $M-D$ smallest GEVs. For brevity, we denote as $\bar{E}$ the $M \times(M-D)$ matrix consisting of these GEVs. Motivated by eqn. 11 , we conduct the following matrix conversion

$$
\bar{E}=\left[\begin{array}{cccc}
g_{1} & 0 & \cdots & 0 \\
0 & g_{2} & \cdots & 0 \\
\vdots & \vdots & \ddots & \vdots \\
0 & 0 & \cdots & g_{M-D}
\end{array}\right] T
$$

where $g_{l}, l=1, \ldots, M-D$ are $(D+1) \times 1$ vectors with a unit leading component, $T$ is an $(M-D) \times(M-D)$ nonsingular matrix, and 0 is a scalar. Note that eqn. 12 consists of $M-D$ equations of the form

$$
\overline{\boldsymbol{E}} \overline{\boldsymbol{t}}_{\boldsymbol{l}}=\left[\begin{array}{c}
0 \\
\vdots \\
\boldsymbol{g}_{l} \\
\vdots \\
0
\end{array}\right] \quad l=1, \ldots, M-D
$$

where $\bar{t}_{l}, l=1, \ldots, M-D$ are the columns of $T^{-1}$ as described by

$$
T^{-1}=\left[\bar{t}_{1}\left|\bar{t}_{2}\right| \cdots \mid \bar{t}_{M-D}\right]
$$


There are two noteworthy points in eqn. 13 . First, the $g_{1}$ vectors are unknown. Secondly, each of the equations represents an overdetermined system which does not in general lead to an exact solution for $\bar{t}_{i}$. Nevertheless, invoking the fact that the leading component of $g_{l}$ equals 1 does lead to a unique solution for $t_{l}$ as obtained by

$$
\left[\begin{array}{c}
\bar{E}(1: l,:) \\
\bar{E}(D+l+1: M,:)
\end{array}\right] \overline{t_{l}}=e_{l} \quad l=1, \ldots, M-D
$$

where $M\left(n_{1}: n_{2},:\right)$ denotes a submatrix consisting of the $n_{1}$ th to the $n_{2}$ th row of $M$ and $e_{1}$ is the $l$ th column of the $(M-D) \times(M-D)$ identity matrix. Note that numerical instability may occur if the submatrix on the left-hand side of eqn. 15 is nearly rank-deficient for some $l$. Although there seems no obvious way to assure that this does not happen under severe noise/error corruption, one may argue that under moderately good conditions, $\bar{E}$ is a full-rank matrix well representing the true noise subspace, which is in turn represented by the banded Toeplitz structure in eqn. 11. In such a case, the full rank of the left-hand side of eqn. 15 should be retained, and the conversion in accordance with eqn. 12 should yield $g_{1} \simeq$ $g_{2} \simeq \cdots \simeq g_{M-D} \simeq g$. Now, with the above-obtained $\bar{t}_{1}$, $l=1, \ldots, M-D$, we can compute $g_{l}, l=1, \ldots, M-D$

$$
\boldsymbol{g}_{l}=\overline{\boldsymbol{E}}(l: D+l,:) \overline{\boldsymbol{t}}_{l} \quad l=1, \ldots, M-D
$$

Note that under noise/error corruption, $g_{1} \neq g_{2} \neq \cdots \neq$ $\boldsymbol{g}_{M-D}$, as opposed to eqn. 11. In fact, the disparity between these $g_{t}$ vectors reflects the severity of the corruption. Although the banded matrix on the right-hand side of eqn. 12 does not represent the true noise subspace as does eqn. 11 , it does span the same range space as that of $\bar{E}$, as $\boldsymbol{T}$ is nonsingular. As the direct correspondence between the DOAs and the $g$ vector no longer exists, we need to invoke the orthogonality condition between $\bar{E}$ and $A$ in the ideal case, and work with the weighted element space null spectrum

$$
S(u)=\boldsymbol{a}^{\boldsymbol{H}}(u) \bar{E} \boldsymbol{P} \bar{E}^{H} \boldsymbol{a}(u)
$$

which, by eqn. 12 , can be rewritten as

$$
S(u)=a_{D}^{H}(u) F D^{*}(u) \boldsymbol{T P}^{H} \boldsymbol{D}(u) \boldsymbol{F}^{\boldsymbol{H}} \boldsymbol{a}_{D}(u)
$$

where

$$
\begin{aligned}
& \boldsymbol{a}_{D}(u)=\left[1, e^{j \pi u}, \ldots, e^{j D \pi u}\right]^{T} \\
& \boldsymbol{F}=\left[\boldsymbol{g}_{1}\left|\boldsymbol{g}_{2}\right| \cdots \mid \boldsymbol{g}_{M-D}\right]
\end{aligned}
$$

and

$$
\boldsymbol{D}(u)=\left[\begin{array}{cccc}
1 & 0 & \cdots & 0 \\
0 & e^{j \pi u} & \cdots & 0 \\
\vdots & \vdots & \ddots & \vdots \\
0 & 0 & \cdots & e^{j(M-D-1) \pi u}
\end{array}\right]
$$

A characteristic feature in eqn. 18 is that the array manifold vector $a_{D}(u)$ involved is $(D+1) \times 1$, instead of $M \times 1$ as in eqn. 9. Note that, except for the $(M-D) \times(M-D)$ diagonal matrix $D(u)$, the null spectrum in eqn. 18 is essentially that associated with a $(D+1)$-element LES array. To fully exploit this reduction in dimension, it is natural to replace $D(u)$ with a constant matrix by fixing $u=u_{o}$, and rewrite eqn. 18 as

$$
S\left(u \mid u_{o}\right)=\boldsymbol{a}_{D}^{H}(u) \boldsymbol{F} \boldsymbol{D}^{*}\left(u_{o}\right) \boldsymbol{T P} \boldsymbol{T}^{H} \boldsymbol{D}\left(u_{o}\right) \boldsymbol{F}^{H} \boldsymbol{a}_{D}(u)
$$

IEE PROCEEDINGS-F, Vol. 139, No.4, AUGUST 1992
Converting back into element space formation, we obtain

$$
\begin{aligned}
S\left(u \mid u_{o}\right)= & a_{D}^{H}(u) \boldsymbol{F} D^{*}(u) \boldsymbol{T} T^{-1} \boldsymbol{D}(u) \boldsymbol{D}^{*}\left(u_{o}\right) \\
& \times \boldsymbol{T P} T^{H} \boldsymbol{D}\left(u_{o}\right) \boldsymbol{D}^{*}(u)\left(\boldsymbol{T} \boldsymbol{T}^{-1}\right)^{H} \boldsymbol{D}(u) \boldsymbol{F}^{H} \boldsymbol{a}_{D}(u) \\
= & \boldsymbol{a}^{\boldsymbol{H}}(u) \bar{E} \boldsymbol{T}^{-1} \boldsymbol{D}\left(u-u_{o}\right) \\
& \times \boldsymbol{T P} \boldsymbol{T}^{H} \boldsymbol{D}^{*}\left(u-u_{o}\right)\left(\boldsymbol{T}^{-1}\right)^{\boldsymbol{H}} \bar{E}^{H} \boldsymbol{a}(u) \\
= & \boldsymbol{a}^{\boldsymbol{H}}(u) \bar{E} \boldsymbol{P}_{o}(u) \bar{E}^{H} \boldsymbol{a}(u)
\end{aligned}
$$

where

$$
P_{o}(u)=T^{-1} D\left(u-u_{o}\right) T P T^{H} D^{*}\left(u-u_{o}\right)\left(T^{-1}\right)^{H}
$$

Note that we have used $D^{*}(u) D(u)=I$ and $D(u) D^{*}\left(u_{o}\right)=$ $D\left(u-u_{o}\right)$. We see from eqn. 23 that replacing $D(u)$ with $\boldsymbol{D}\left(u_{o}\right)$ is, in fact, tantamount to replacing $\boldsymbol{P}$ with an angledependent weighting matrix $\boldsymbol{P}_{0}(u)$. Since $\boldsymbol{P}_{0}(u)$ is positivesemidefinite, the modified null spectrum eqn. 23 will provide the true DOAs under no noise/error condition In practical noisy cases, the estimation accuracy may degrade using eqn. 23 if $\boldsymbol{P}_{o}(u)$ differs greatly from $\boldsymbol{P}$ for $u \simeq u_{i}, i=1, \ldots, D[10]$. To gain further insight, consider the orthogonality conditions under no noise/error

$$
\begin{aligned}
S\left(u_{i}\right) & =\boldsymbol{a}^{\boldsymbol{H}}\left(u_{i}\right) \overline{\boldsymbol{E}} \boldsymbol{P} \overline{\boldsymbol{E}}^{\boldsymbol{H}} \boldsymbol{a}(u) \\
& =\boldsymbol{a}_{\boldsymbol{D}}^{\boldsymbol{H}}\left(u_{i}\right) \boldsymbol{F} \boldsymbol{D}^{\boldsymbol{*}}\left(u_{i}\right) \boldsymbol{T} \boldsymbol{P} \boldsymbol{T}^{\boldsymbol{H}} \boldsymbol{D}\left(u_{i}\right) \boldsymbol{F}^{\boldsymbol{H}} \boldsymbol{a}_{D}\left(u_{i}\right) \\
& =0 \quad i=1, \ldots, D
\end{aligned}
$$

Comparing eqns. 22 and 24 , it is evident that in order to achieve the performance of element space eigen-based methods working with $P$, we must choose $u_{o} \simeq u_{i}$ in estimating $u_{i}$. In short, to retain the feature of reduceddimension null spectrum and the efficacy of eigen-based processing, a set of fixing angles $\hat{u}_{i}^{0} \simeq u_{i}, i=1, \ldots, D$ must be chosen. However, this requires the knowledge of the DOAs that we are trying to estimate, and thus suggests an iterative procedure wherein we begin with the formation of the $D$ reduced-dimension null spectra

$$
\begin{array}{r}
S\left(u \mid \hat{u}_{i}^{0}\right)=a_{D}^{H}(u) F D^{*}\left(\hat{u}_{i}^{0}\right) T P T^{H} D\left(\hat{u}_{i}^{0}\right) F^{H} a_{D}(u) \\
i=1, \ldots, D
\end{array}
$$

A new set of DOA estimates $\hat{u}_{i}^{1}, i=1, \ldots, D$ is obtained as the solutions to $S\left(u \mid \hat{u}_{i}^{0}\right)=0, i=1, \ldots, D$, respectively, corresponding to the first iteration. To exploit the high resolution of root-form methods, these are done by rooting the following $2 D$ th-order reduced-order signal polynomials

$$
\begin{array}{r}
S\left(z \mid \hat{u}_{i}^{0}\right)=\boldsymbol{v}_{D}^{T}\left(z^{-1}\right) \boldsymbol{F} \boldsymbol{D}^{*}\left(\hat{u}_{i}^{0}\right) \boldsymbol{T P} \boldsymbol{T}^{H} \boldsymbol{D}\left(\hat{u}_{i}^{0}\right) \boldsymbol{F}^{H} \boldsymbol{v}_{D}(z) \\
i=1, \ldots, D
\end{array}
$$

where $v_{D}(z)=\left[1, z, \ldots, z^{D}\right]^{T}$. Note that the coefficients of $S\left(z \mid \hat{u}_{i}^{0}\right)$ exhibit conjugate centrosymmetry, such that the corresponding $2 D$ roots form $D$ conjugate reciprocal pairs. That is, if $z_{o}$ is a root of $S\left(z \mid \hat{u}_{i}^{0}\right)$, then so is $1 / z_{o}^{*}$. As a consequence, only $D$ distinct values are observed regarding the phase angles of the $2 D$ roots. Specifically, we choose $\hat{u}_{i}^{1}$ as that phase angle closest to $\hat{u}_{i}^{0}$. It should be pointed out that, in the above, only a single DOA estimate is extracted from each reduced-order signal polynomial. A new set of reduced-order signal polynomials $S\left(z \mid \hat{u}_{i}^{1}\right), i=1, \ldots, D$ is then formed, based on $\hat{u}_{i}^{1}$, $i=1, \ldots, D$. The procedure is iterated until the DOA estimates converge. The execution of the algorithm is concurrent in nature, making parallel processing a suitable way of implementation.

The above procedure converts the original problem of determining $D$ roots from a $2(M-1)$ th-order polynomial 
into $D$ parallel problems, each of determining a single root from a $2 D$ th-order polynomial. Unfortunately, the reduction of the polynomial order from $2(M-1)$ to $2 D$ requires that the full element-space noise subspace is available, so that the conversion in accordance with eqn. 12 is possible. This, in turn, requires the full $M \times M$ eigenanalysis of the element-space data/noise correlation matrix pencil. The heavy computational load associated with eigenanalysis necessitates the previously stated beamspace transformation.

\subsection{Beamspace implementation}

Recall that in beamspace eigen-based procedures, the vectors available that lie in the element-space noise subspace are those $K-D$ columns of $\bar{E}_{W}$ as given in eqn. 9 . As in eqn. 12, we conduct the following matrix conversion

$$
\bar{E}_{W}=\left[\begin{array}{cccc}
\boldsymbol{h}_{1} & 0 & \cdots & 0 \\
0 & \boldsymbol{h}_{2} & \cdots & 0 \\
\vdots & \vdots & \ddots & \vdots \\
0 & 0 & \cdots & \boldsymbol{h}_{K-D}
\end{array}\right] U
$$

where $h_{l}, l=1, \ldots, K-D$ are $(M-K+D+1) \times 1$ vectors with unit leading components, $U$ is a $(K-D) \times(K-D)$ nonsingular matrix, and 0 is a scalar. Note that since $\bar{E}_{W}$ has only $K-D$ columns, it cannot be converted into a banded form with bandwidth $D+1$ unless $K=M$ and/or SNR $=\infty$. Direct implementation of the iterative procedure prescribed in eqn. 13-26, based on $\bar{E}_{W}$, then requires rooting in parallel $D 2(M-K+D)$ th-order polynomials at each iteration Summarising at this point, the general computational requirement includes a $K \times K$ GEVD and the rooting of several $2(M-K+D)$ th-order polynomials. This is in contrast to that associated with element-space implementation: an $M \times M$ GEVD and the rooting of several $2 D$ th-order polynomials. In fact, for $M \gg K$, the conversion in accordance with eqn. 27 , along with the subsequent iterations, is an overaction. In other words, the simplification due to reduced-dimension eigenanalysis is offset by the increased complexity due to higher-order polynomial rooting. To take full advantage of working with the reduced-dimension beamspace eigenanalysis and the reduced-order signal polynomials, a complete noise subspace of dimension $M-D$ must be estimated, based on the beamspace data. To this end, we propose the concept of noise subspace augmentation. The augmentation is done by appending $M-K$ columns to $\bar{E}_{W}$, to obtain an $M \times(M-D)$ matrix representing the full noise subspace.

\subsection{Noise subspace augmentation}

Consider appending to $\overline{\boldsymbol{E}}_{W}$ an $M \times(M-K)$ matrix $\bar{E}_{X}$ to form an $M \times(M-D)$ matrix $\bar{E}_{A V G}$

$$
\bar{E}_{A U G}=\left[\bar{E}_{W} \mid \bar{E}_{X}\right]
$$

$\bar{E}_{X}$ is so selected that $\bar{E}_{A U G}$ represents the noise subspace to a good approximation, i.e. $\bar{E}_{A U G}^{H} \boldsymbol{A} \simeq \mathbf{0}$. Clearly $\widetilde{\boldsymbol{E}}_{X}$ depends on $A$ and must thus be determined from a set of DOA estimates. This, again, suggests an iterative implementation wherein we refine $\bar{E}_{A U G}$ each time a new set of DOA estimates is obtained. Suppose that preliminary DOA estimates $\hat{u}_{i}^{0}, i=1, \ldots, D$, and a corresponding DOA matrix estimate $\hat{A}$ are available, we may choose $E_{X}$ to be composed of the $M-K$ basis vectors for $\operatorname{Null}\left\{\left[\bar{E}_{W} \mid \hat{A}^{H}\right]^{H}\right.$. Although this approach ensures that the columns of the resulting $\bar{E}_{A U G}$ form a linearly independ- ent set, we do not recommend it, because of its numerical complexity. As an alternative, we suggest the following much simpler implementation.

With $\hat{u}_{i}^{0}, i=1, \ldots, D$, we can form a $D$ th-order polynomial $g^{0}(z)=\sum_{m=0}^{D}\left(\gamma_{m}^{0}\right)^{*} z^{m}$, with $\gamma_{0}^{0}=1$, whose roots are $z_{i}^{0}=e^{j \pi a_{i}^{0}}, i=1, \ldots, D$. Let $g^{0}=\left[1, \gamma_{1}^{0}, \ldots, \gamma_{D}^{0}\right]^{T}$ be the $(D+1) \times 1$ coefficient vector associated with $g^{0}(z)$. According to eqn. 11 , the columns of the $M \times(M-D)$ matrix

$$
\boldsymbol{G}^{\mathbf{0}}=\left[\begin{array}{cccc}
\boldsymbol{g}^{\mathbf{0}} & 0 & \cdots & 0 \\
\mathbf{0} & \boldsymbol{g}^{\mathbf{0}} & \cdots & 0 \\
\vdots & \vdots & \ddots & \vdots \\
\mathbf{0} & 0 & \cdots & \boldsymbol{g}^{\mathbf{0}}
\end{array}\right]
$$

form the basis of $\operatorname{Null}\left\{\hat{A}^{H}\right\}$ and approximately span the full element-space noise subspace, as long as the preliminary DOA estimates are not too erroneous. In such a case, any set of $M-K$ columns of $G^{0}$ may be chosen to constitute $E_{X}$. However, care must be taken to ensure that the resulting $\bar{E}_{A U G}$ is full-rank, to avoid numerical instability in the subsequent development. An empirical criterion would be to check if any $K-D$ rows of $\bar{E}_{W}$ nearly form a linearly dependent set. If this happens, we should not append all zeros to these rows. For simplicity, we assume that the first $K-D$ rows of $\bar{E}_{W}$ are linearly independent and choose $E_{X}=G^{0}(:, K-D+1: M-D)$, where $M\left(:, n_{1}: n_{2}\right)$ denotes a submatrix composed of the $n_{1}$ th to $n_{2}$ th column of $M$. The augmented noise EV matrix now has the following form

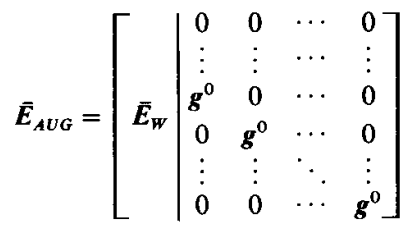

With the $\bar{E}_{A U G}$ obtained in eqn. 30 and the procedure outlined in Section 3.1, we find a $(M-D) \times(M-D)$ nonsingular matrix $T$, such that

$$
\overline{\boldsymbol{E}}_{A U G}=\left[\begin{array}{cccc}
\boldsymbol{g}_{1} & 0 & \cdots & 0 \\
0 & \boldsymbol{g}_{2} & \cdots & 0 \\
\vdots & \vdots & \ddots & \vdots \\
0 & 0 & \cdots & \boldsymbol{g}_{M-D}
\end{array}\right] \boldsymbol{T}
$$

where $\boldsymbol{T}$ is determined by

$$
\left[\begin{array}{c}
\bar{E}_{A U G}(1: l,:) \\
\bar{E}_{A U G}(D+l+1: M,:)
\end{array}\right] \bar{t}_{l}=e_{l} \quad l=1, \ldots, M-D
$$

The various vectors $g_{l}, \bar{t}_{l}$ and $e_{l}, l=1, \ldots, M-D$ are as previously defined. Comparing eqns. 30 and 31 , it is easily seen that $\boldsymbol{T}^{-1}$ has the following form

$$
T^{-1}=\left[\begin{array}{cc}
\Sigma & 0 \\
\Gamma & I_{M-K}
\end{array}\right]
$$

where $\Sigma$ is $(K-D) \times(K-D), \Gamma$ is $(M-K) \times(K-D)$ $O$ is the $(K-D) \times(M-K)$ zero matrix, and $I_{M-K}$ is the $(M-K) \times(M-K)$ identity matrix. The substitution of eqns. 31 and 33 into eqn. 30 yields

$$
g_{l}=g^{0} \quad l=K-D+1, \ldots, M-D
$$

To obtain $\Sigma, \Gamma$ and $g_{l}, l=1, \ldots, K-D$ we need only to solve the first $K-D$ equations in eqn. 32. Now, comparing the corresponding columns in eqns. 30 and 31 , and

IEE PROCEEDINGS-F, Vol. 139, No. 4, AUGUST 1992 
using eqns. 33-34, we have

$$
\overline{\boldsymbol{E}}_{\boldsymbol{W}}=\left[\begin{array}{cccccc}
\boldsymbol{g}_{1} & \cdots & 0 & 0 & \cdots & 0 \\
\vdots & \ddots & \vdots & \vdots & \ddots & \vdots \\
0 & \cdots & \boldsymbol{g}_{\mathbf{K}-D} & 0 & \cdots & 0 \\
0 & \cdots & 0 & \boldsymbol{g}^{0} & \cdots & 0 \\
\vdots & \ddots & \vdots & \vdots & \ddots & \vdots \\
0 & \cdots & 0 & 0 & \cdots & \boldsymbol{g}^{0}
\end{array}\right] \boldsymbol{T}_{W}
$$

where $\boldsymbol{T}_{W}$ consists of the first $K-D$ columns of $T$

$$
T_{W}=\left[\begin{array}{c}
\Sigma^{-1} \\
-\Gamma \Sigma^{-1}
\end{array}\right]
$$

Note that, as opposed to eqn. 12 , the same $g^{0}$ vector is used to form the right submatrix in eqn. 35 . This represents an approximation under the noise/error corruption due to eqn. 30. The development up to this point has converted $\bar{E}_{W}$ into a banded form, with bandwidth $D+1$, as was originally achieved with element-space implementation. Now, substituting eqn. 35 into eqn. 9, and following the manipulations in eqns. 17-21, we obtain

$$
\begin{aligned}
S_{B}(u) & =a^{H}(u) \bar{E}_{W} P \bar{E}_{W}^{H} a(u) \\
& =a_{D}^{H}(u) F_{W} D^{*}(u) T_{W} P T_{W}^{T} D(u) F_{W}^{H} a_{D}(u)
\end{aligned}
$$

where $a_{D}(u)$ and $D(u)$ are given by eqns. 19 and 21 , respectively, and

$$
\boldsymbol{F}_{W}=\left[\boldsymbol{g}_{1}|\cdots| \boldsymbol{g}_{K-D}\left|\boldsymbol{g}^{0}\right| \cdots \mid \boldsymbol{g}^{0}\right]
$$

To work with a $(D+1) \times 1$ manifold vector, we need to fix the angle $u$ in $D(u)$. Following the development in Section 3.1, we first choose a set of preliminary DOA estimates $\hat{u}_{i}^{0}, i=1, \ldots, D$. These angles are available from the earlier stage of noise subspace augmentation. We then construct a set of reduced-dimension null spectra

$$
\begin{array}{r}
S_{B}\left(u \mid \hat{u}_{i}^{0}\right)=a_{D}^{H}(u) F_{W} D^{*}\left(\hat{u}_{i}^{0}\right) T_{W} P T_{W}^{H} D\left(\hat{u}_{i}^{0}\right) F_{W}^{H} a_{D}(u) \\
i=1, \ldots, D
\end{array}
$$

A new set of DOA estimates $\hat{u}_{i}^{1}, i=1, \ldots, D$ is obtained by solving $S_{\mathrm{B}}\left(u \mid \hat{u}_{i}^{0}\right)=0, i=1, \ldots, D$, respectively, corresponding to the first iteration. Again, these are done by rooting the following $2 D$ th order reduced-order signal polynomials

$$
\begin{array}{r}
S_{B}\left(z \mid \hat{u}_{i}^{0}\right)=v_{D}^{T}\left(z^{-1}\right) F_{W} D^{*}\left(\hat{u}_{i}^{0}\right) T_{W} P T_{W}^{H} \boldsymbol{D}\left(\hat{u}_{i}^{0}\right) \boldsymbol{F}_{W}^{H} v_{D}(z) \\
i=1, \ldots, D
\end{array}
$$

The signal roots are selected in the same way as suggested for element-space implementation. Based on the new DOA estimates, we can proceed to perform noise subspace augmentation, which, in turn, produces a new set of reduced-order signal polynomials. The procedure is iterated until the DOA estimates converge.

In summary, beamspace transformation reduces the dimension of the eigenanalysis from $M \times M$ to $K \times K$ whereas noise subspace augmentation, along with banded form conversion, reduces the order of the signal polynomial from $2(M-1)$ to $2 D$. Each iteration of the algorithm is concurrent in nature, in that both the rooting of the reduced-order signal polynomials and the banded form conversion involved can be executed in parallel. This helps to alleviate the extra complexity due to eqns. $30-36$.

3.4 Determination of preliminary DOA estimates Good preliminary DOA estimates are essential to the above new method. As we are mainly interested in detecting closely spaced sources, the effective angular passband of the beamformer is typically narrow, to minimise

IEE PROCEEDINGS-F, Vol. 139, No. 4, AUGUST 1992 out-of-band noise power. Suppose that the passband of the beamformer is $\left[u_{L}, u_{R}\right]$, where $u_{L}<u_{i}<u_{R}, i=1$, $\ldots, D$. Without any further information about the DOAs, a natural guess is that $u_{i}=\bar{u}=\frac{1}{2}\left(u_{L}+u_{R}\right), i=1, \ldots, D$. With these coarsely guessed DOAs, a set of preliminary DOA estimates can be obtained via the execution of the algorithm outlined in the previous section, for a single iteration. In so doing, we simply set $\hat{u}_{i}^{0}=\bar{u}, i=1, \ldots, D$, and replace $g^{0}$ in eqn. 29 by $\bar{g}$, the coefficient vector of $\bar{g}(z)=\left(z-e^{j \pi \bar{u}}\right)^{D}$. A reduced-order signal polynomial $S_{B}(z \mid \bar{u})$ is then constructed according to eqn. 40 . Note that in this case there is only one polynomial to work with. Finally, the preliminary DOA estimates are determined from the $D$ roots $\bar{z}_{i}, i=1, \ldots, D$, of $S_{B}(z \mid \bar{u})$, according to $\hat{u}_{i}^{0}=(1 / \pi) \arg \left\{\bar{z}_{i}\right\}, i=1, \ldots, D$.

\subsection{Convergence behaviour of the algorithm}

The convergence behaviour of the new algorithm can be described as follows. As demonstrated by eqn. 23, fixing the angle in $\boldsymbol{D}(u)$, or replacing the true null spectrum by a reduced-dimension one is, in fact, equivalent to replacing $\boldsymbol{P}$ by another positive-semidefinite angle-dependent weighting matrix. It follows that each of the reduceddimension null spectra in eqn. 39 , or each of the reducedorder signal polynomials in eqn. 40 , will provide the true DOAs under no noise/error condition. In practical noisy cases, the estimation accuracy may degrade, as the new weighting matrix may not be optimum compared to $\boldsymbol{P}$. Nevertheless, the degradation is insignificant if the fixing angles are close to the true DOAs. We conclude that the general situation is that good DOA estimates help to improve the reduced-order signal polynomials, and vice versa. Hence, so long as preliminary DOA estimates are not too erroneous and the SNR is not too low, the new DOA estimates should approach the DOAs obtained with the true signal polynomial as the iterations proceed. It should be pointed out that the final DOA estimates upon convergence are not exactly those obtained with the true signal polynomial. This is due to the fact that the signal roots associated with the true signal polynomial may not lie on the unit circle, but the conversion from eqn. 39 to eqn. 40 inherently assumes that all signal roots are on the unit circle. However, the disparity is insignificant if the SNR is not too low.

Simulations show that, under moderate conditions, the algorithm typically converges in 4-5 iterations, with a precision of $10^{-3}$ beamwidth of the array. The behaviour of the algorithm under severe noise/error corruption is not clearly predictable. It is found by simulations that the DOA estimates still converge at a very low SNR, but it takes more iterations to achieve it. We do not here intend to tackle the problem of extremely low SNR, since eigenbased methods are known to perform poorly under such conditions [10].

\subsection{Selection of weighting matrix}

The selection of the weighting matrix $P$ greatly affects the performance of the corresponding DOA estimator. Twocommonly employed beamspace root-form eigen-based methods - beamspace root-MUSIC and beamspace root-minimum-norm - correspond to $\boldsymbol{P}=\boldsymbol{I}$ and $\boldsymbol{P}=\boldsymbol{c} c^{H}$, respectively, where $c$ denotes the transpose of the first row of $\bar{E}_{W}$. Statistical performance analysis [4] shows that the mean-squared error of the DOA estimates associated with root-minimum-norm is lower-bounded by that associated with root-MUSIC, with the disparity increasing as the number of elements $M$ increases. The superiority of root-MUSIC over root-minimum-norm, 
however, is overshadowed by the issue of numerical complexity. Note that root-MUSIC requires rooting a $2(M-1)$ th-order polynomial, whereas root-minimumnorm requires only rooting a $(M-1)$ th-order one. The problem of excessive computations associated with rootMUSIC with large $M$ is greatly alleviated by invoking the proposed idea of polynomial order reduction. By order reduction, root-MUSIC would require rooting in parallel $D 2 D$ th-order polynomials at each iteration, whereas root-minimum-norm requires only rooting $D$ th order ones. For small $D$, the difference between the numerical complexity associated with these two methods is negligible, as opposed to the case without order reduction. Hence, for the case of large $M$ and small $D$, it is recommended that root-MUSIC be used in conjunction with order reduction.

As an alternative, the weighting matrix may be considered as acting on the $(D+1) \times(K-D)$ reduceddimension noise $\mathrm{EV}$ matrix $\boldsymbol{E}_{\mathrm{D}}\left(\hat{\boldsymbol{u}}_{\mathrm{i}}^{k}\right)$ as follows

$$
\begin{array}{r}
S_{B}\left(z \mid \hat{u}_{i}^{k}\right)=v_{D}^{T}\left(z^{-1}\right) E_{D}\left(\hat{u}_{i}^{k}\right) P_{i}^{k} E_{D}^{H}\left(\hat{u}_{i}^{k}\right) v_{D}(z) \\
i=1, \ldots, D
\end{array}
$$

where

$$
\boldsymbol{E}_{\mathrm{D}}\left(\hat{u}_{i}^{k}\right)=\boldsymbol{F}_{W} \boldsymbol{D}^{*}\left(\hat{u}_{i}^{k}\right) \boldsymbol{T}_{W} \quad i=1, \ldots, D
$$

We denote the weighting matrix in eqn. 41 as $P_{i}^{k}$ to emphasise its dependence upon $\boldsymbol{E}_{\mathrm{D}}\left(\hat{u}_{i}^{k}\right)$. That is, we may use different weighting matrices for different sources at different iterations. In this case, we treat $S_{B}\left(z \mid \hat{u}_{i}^{k}\right)$ as the signal polynomial associated with the noise EV matrix $E_{D}\left(\hat{u}_{i}^{k}\right)$ generated by the data obtained from a $(D+1)$ element LES array. Applications of root-MUSIC and root-minimum-norm from such a viewpoint correspond to $\boldsymbol{P}_{i}^{k}=\boldsymbol{I}$ and $\boldsymbol{P}_{i}^{k}=\boldsymbol{c}_{D}\left(\hat{u}_{i}^{k}\right) \boldsymbol{c}_{D}^{H}\left(\hat{\boldsymbol{u}}_{i}^{k}\right)$, respectively, where $\boldsymbol{c}_{D}\left(\hat{u}_{i}^{k}\right)$ is the transpose of the first row of $E_{D}\left(\hat{u}_{i}^{k}\right)$. Note that rootMUSIC remains unchanged, whereas root-minimumnorm requires a different weighting matrix for each reduced-order signal polynomial at each iteration. We now briefly discuss the effect of working with the reduced-dimension noise EV matrices. We first observe from eqn. 38 that, upon the convergence of the algorithm, $\boldsymbol{E}_{D}\left(\hat{u}_{i}^{k}\right)$ is approximately rank-one, since $\boldsymbol{g}_{1} \simeq \cdots \simeq$ $\boldsymbol{g}_{\boldsymbol{K}-\boldsymbol{D}} \simeq \boldsymbol{g}^{\boldsymbol{k}}$. This implies that upon the convergence of the algorithm, $\boldsymbol{E}_{D}\left(\hat{u}_{i}^{k}\right) \boldsymbol{E}_{D}^{H}\left(\hat{u}_{i}^{k}\right)$ is approximately equal to $\boldsymbol{E}_{D}\left(\hat{u}_{i}^{k}\right) \boldsymbol{c}_{D}\left(\hat{u}_{i}^{k}\right) \boldsymbol{c}_{D}^{H}\left(\hat{u}_{i}^{k}\right) \boldsymbol{E}_{D}^{H}\left(\hat{u}_{i}^{k}\right)$ up to a scalar multiple. Hence, both root-MUSIC and root-minimum-norm applied in this manner should yield approximately the same DOA estimates upon the convergence of the algorithm. This is confirmed by simulation results. The advantage of working with root-minimum-norm is that only $D$ th-order polynomial rooting is required at each iteration.

\subsection{Algorithm summary}

An outline of the above proposed beamspace DOA estimation procedure is as follows

(i) Collect the beamspace data snapshot vectors $x_{B}[n]=W^{H} x[n], n=1, \ldots, N$; form the beamspace data correlation matrix

$$
\hat{R}_{B B}=\frac{1}{N} \sum_{n=1}^{N} x_{B}[n] x_{B}^{H}[n]
$$

where $W$ is the $M \times K$ beamforming matrix, and determine the beamspace noise correlation matrix $\boldsymbol{Q}_{B B}$

(ii) Perform the GEVD of the $K \times K$ pencil $\left\{\hat{\boldsymbol{R}}_{B B}\right.$, $\left.Q_{B B}\right\}$. Let $\bar{E}_{B}$ be composed of the $K-D$ GEVs corresponding to the $K-D$ smallest GEVs. Let $\bar{E}_{W}=W \bar{E}_{B}$.
Obtain the preliminary DOA estimates $\hat{u}_{i}^{0}, i=1, \ldots, D$, according to Section 3.4. Initialise with $k=0$.

(iii) With the DOA estimates $\hat{u}_{i}^{k}, i=1, \ldots, D$, execute the procedure described by eqns. $29-36$ to obtain the decomposition of $\bar{E}_{W}$ described by eqn. 35 .

(iv) Construct the reduced-order signal polynomials $S_{B}\left(z \mid \hat{u}_{i}^{k}\right), i=1, \ldots, D$ in accordance with eqn. 40 . Select the weighting matrix

\section{Root-MUSIC: $\boldsymbol{P}=\boldsymbol{I}$}

Root-minimum-norm-1: $\boldsymbol{P}=\boldsymbol{c} c^{H}$

Root-minimum-norm-2: $\boldsymbol{P}=\boldsymbol{P}_{i}^{k}=\boldsymbol{c}_{D}\left(\hat{u}_{i}^{k}\right) \boldsymbol{c}_{D}^{H}\left(\hat{u}_{i}^{k}\right)$

where $c$ is the transpose of the first row of $\bar{E}_{W}$ and $c_{D}\left(\hat{u}_{i}^{k}\right)$ is the transpose of the first row of $E_{D}\left(\hat{u}_{i}^{k}\right)$, as defined by eqn. 42.

(v) Root $S_{B}\left(z \mid \hat{u}_{i}^{k}\right), i=1, \ldots, D$ in parallel. Among these roots, determine a new set of DOA estimates $\hat{u}_{i}^{k+1}, i=1$, $\ldots, D$, according to Section 3.1 .

(vi) Check the convergence of the DOA estimates. If yes, terminate the algorithm. Otherwise, go back to (iii), with $\hat{u}_{i}^{k}$ replaced by $\hat{u}_{i}^{k+1}, i=1, \ldots, D$.

\section{Computer simulations}

Computer simulations were conducted to demonstrate the performance of the proposed methods. The array employed consisted of 33 identical elements equally spaced by a half wavelength. The source signal was modelled as a Gaussian random process with zero mean and a variance of $\sigma_{s}^{2}$. The noise present at the array elements was assumed to be spatially white zero-mean Gaussian, with power $\sigma_{n}^{2}$ such that the beamspace noise correlation matrix is given by $Q_{B B}=\sigma_{n}^{2} W^{H} W$. The SNR in $\mathrm{dB}$ was defined as $10 \log _{10}\left(\sigma_{s}^{2} / \sigma_{n}^{2}\right)$

The first set of simulations compared the performance of the various root-form eigen-based methods presented in the paper. For all cases, $N=50$ snapshots were collected at each trial to obtain the DOA estimates, and 50 independent Monte-Carlo trials were executed to obtain the sample statistics. For brevity, we refer to the original and the proposed versions of the beamspace rootMUSIC method as rt-MUSIC ${ }_{M}$ and rt-MUSIC ${ }_{D}$, respectively. Note that the subscripts indicate the respective dimensions involved in the two methods. Likewise, we refer to the original and the two proposed versions of the beamspace root-minimum-norm method, as described in the algorithm summary, as rt- $\mathrm{MN}_{M}, \mathrm{rt}-\mathrm{MN}_{D^{-1}}$ and rt- $\mathrm{MN}_{D}-2$ respectively. The scenario involved three uncorrelated equal-power sources at $7^{\circ}, 8.5^{\circ}$ and $12^{\circ}$ with respect to the broadside of the array. The angular separation between the first two sources was $1.5^{\circ}$, which is less than one-half the 3-dB beamwidth of the array $\left(\simeq 3.5^{\circ}\right)$ The matrix beamformer employed was composed of five Chebyshev beams with $-25 \mathrm{~dB}$ sidelobes pointed at $5^{\circ}$, $7^{\circ}, 9^{\circ}, 11^{\circ}$ and $13^{\circ}$. Fig. 1 shows the superposition of these beam patterns. For this particular beamformer, the initial guesses for the DOAs were chosen to be $\bar{u}=$ $\sin \left(9^{\circ}\right)$. The algorithms were terminated if all three DOA estimates converged within $0.01^{\circ}$. The respective sample means and sample standard deviations of the DOA estimates obtained with the aforementioned methods for three different SNR levels are listed in Table 1. Observing the proximity among the results obtained with rt-MUSIC $_{M}$, rt-MUSIC ${ }_{D}$ and rt-MN ${ }_{D}-2$ confirms our earlier claim that both rt-MUSIC ${ }_{D}$ and $\mathrm{rt}-\mathrm{MN}_{D}-2$ should perform comparably to rt-MUSIC ${ }_{M}$. Similar observations were made regarding rt- $M N_{M}$ and rt-MN ${ }_{D}-1$. Note 
that, for this simulation, root-MUSIC and rootminimum-norm yield similar results, as the number of noise EVs is relatively small. To evaluate the convergence rates of the proposed methods, the average number of iterations executed per trail was listed for each case.
For each acquisition, the two beams were steered simultaneously via progressive phase-shifting to a position for which their bisector angle (boresight angle) was closest to the target angle. Note that, in this particular tracking environment, the preliminary angle estimate for the

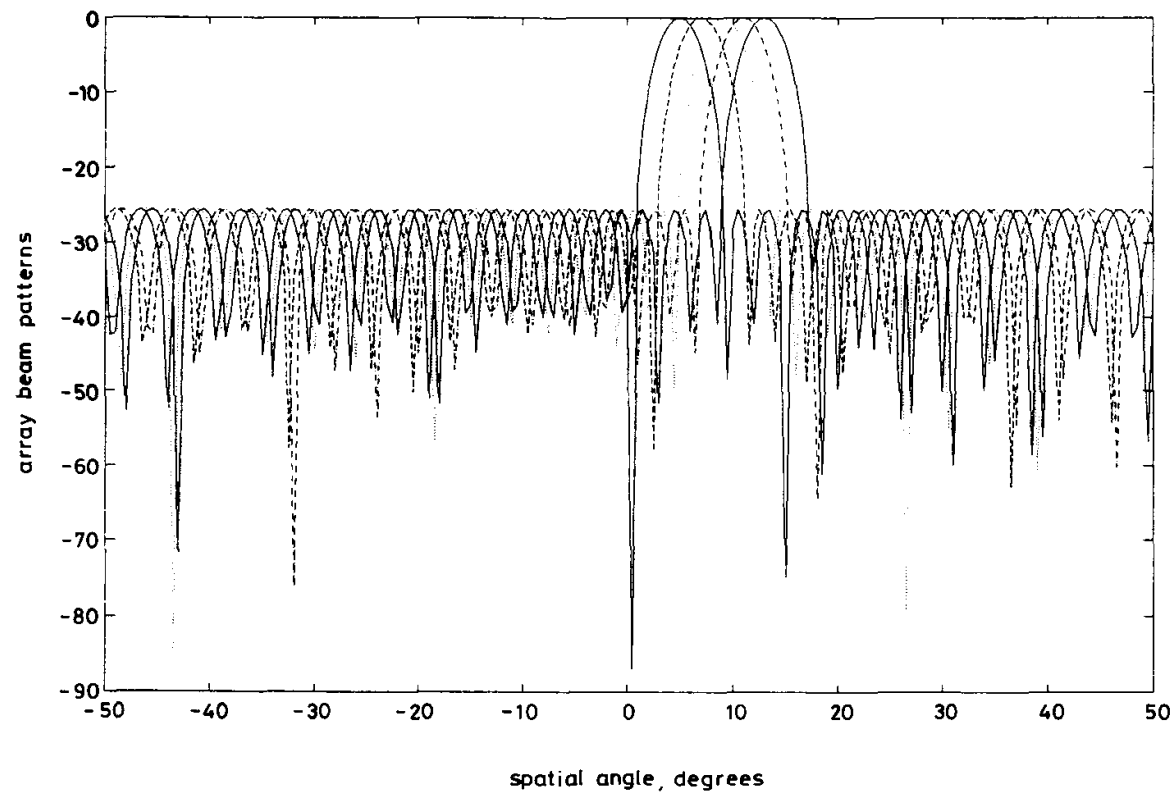

Fig. 1 Superposition of $K=5$ beampatterns (in $d B$ ) generated by an $M=33$-element LES array employing Chebyshev weighting with $-25 d B$ sidelobes

Impressively, all three methods require fewer than five iterations to converge.

The second set of simulations demonstrates the efficacy of the proposed methods applied to monopulse bearing estimation. Two Chebyshev beams with $-25 \mathrm{~dB}$ sidelobes were formed in the vicinity of a single target flying above the array. The target angle varied from $0^{\circ}$ to $45^{\circ}$, with a step size of $1^{\circ}$. The two beams were steered to effectively illuminate the targets. To take into account the practical limitation of analogue beamforming, the minimum step size of the beamsteering was set to be $4^{\circ}$. present acquisition can be simply chosen to be the angle estimate from the previous acquisition. The target echoto-noise ratio was $10 \mathrm{~dB}$ and $N=1$ snapshot corresponding to the monopulse was used for each acquisition. The simulation was initialised with the preliminary angle estimate $\theta^{0}=\sin ^{-1}\left(u^{0}\right)=-1^{\circ}$, and the initial pointing angles of the two beams were set to be $-1.5^{\circ}$ and $1.5^{\circ}$, respectively. For each acquisition, the proposed $\mathrm{rt}-\mathrm{MN}_{D}-2$ algorithm was executed for three iterations to obtain the angle estimate. Fig. 2 shows the trajectory of the resulting angle estimates obtained from 46 acquisi-

\begin{tabular}{|c|c|c|c|c|c|c|c|}
\hline & \multirow[b]{2}{*}{$\begin{array}{l}\text { SNR } \rightarrow \\
\hat{\theta}_{1}, \hat{\sigma}_{1} \\
\hat{\theta}_{2}, \partial_{2} \\
\hat{\theta}_{3}, \hat{\theta}_{3} \\
\hat{\theta}_{1}, \hat{\sigma}_{1} \\
\hat{\theta}_{2}, \hat{\sigma}_{2} \\
\hat{\theta}_{3}, \partial_{3}\end{array}$} & \multicolumn{2}{|c|}{$-5 \mathrm{~dB}$} & \multicolumn{2}{|c|}{$10 \mathrm{~dB}$} & \multicolumn{2}{|c|}{$20 \mathrm{~dB}$} \\
\hline Rt-MUSIC ${ }_{D}$ & & $\begin{array}{r}6.9998 \\
8.5275 \\
11.9896 \\
6.9935 \\
8.5249 \\
11.9909\end{array}$ & $\begin{array}{l}0.3224 \\
0.3954 \\
0.1512 \\
0.3238 \\
0.3968 \\
0.1512\end{array}$ & $\begin{array}{r}7.0024 \\
8.5022 \\
11.9989 \\
7.0023 \\
8.5021 \\
11.9989\end{array}$ & $\begin{array}{l}0.0522 \\
0.0580 \\
0.0238 \\
0.0523 \\
0.0580 \\
0.0238\end{array}$ & $\begin{array}{r}7.0008 \\
8.5008 \\
11.9997 \\
7.0008 \\
8.5008 \\
11.9996\end{array}$ & $\begin{array}{l}0.0165 \\
0.0181 \\
0.0074 \\
0.0165 \\
0.0181 \\
0.0075\end{array}$ \\
\hline NIT & & 4.7 & & 4.7 & & 4.8 & \\
\hline $\mathbf{R t}^{-\mathbf{M N}_{M}}$ & $\begin{array}{l}\hat{\theta}_{1}, \hat{\theta}_{1} \\
\hat{\theta}_{2}, \hat{\sigma}_{2} \\
\hat{\theta}_{3}, \hat{\partial}_{3}\end{array}$ & $\begin{array}{r}7.0037 \\
8.5298 \\
11.9868\end{array}$ & $\begin{array}{l}0.3333 \\
0.4601 \\
0.1538\end{array}$ & $\begin{array}{r}7.0032 \\
8.5034 \\
11.9984\end{array}$ & $\begin{array}{l}0.0541 \\
0.0603 \\
0.0241\end{array}$ & $\begin{array}{r}7.0010 \\
8.5012 \\
11.9995\end{array}$ & $\begin{array}{l}0.0171 \\
0.0188 \\
0.0076\end{array}$ \\
\hline Rt $-\mathbf{M N}_{0}-1$ & $\begin{array}{l}\hat{\theta}_{1}, \partial_{1} \\
\hat{\theta}_{2}, \partial_{2} \\
\hat{\theta}_{3}, \partial_{3}\end{array}$ & $\begin{array}{r}7.0267 \\
8.5216 \\
11.9887\end{array}$ & $\begin{array}{l}0.3207 \\
0.3945 \\
0.1529\end{array}$ & $\begin{array}{r}7.0031 \\
8.5034 \\
11.9985\end{array}$ & $\begin{array}{l}0.0541 \\
0.0602 \\
0.0240\end{array}$ & $\begin{array}{r}7.0010 \\
8.5012 \\
11.9995\end{array}$ & $\begin{array}{l}0.0171 \\
0.0188 \\
0.0076\end{array}$ \\
\hline NIT & & 4 & & 4.8 & & 4.9 & \\
\hline Rt-MN $D_{D}^{-2}$ & $\begin{array}{l}\hat{\theta}_{1}, \partial_{1} \\
\hat{\theta}_{2}, \partial_{2} \\
\hat{\theta}_{3}, \partial_{3}\end{array}$ & $\begin{array}{r}7.0008 \\
8.4990 \\
11.9911\end{array}$ & $\begin{array}{l}0.3368 \\
0.3965 \\
0.1515\end{array}$ & $\begin{array}{r}7.0023 \\
8.5022 \\
11.9987\end{array}$ & $\begin{array}{l}0.0523 \\
0.0580 \\
0.0240\end{array}$ & $\begin{array}{r}7.0008 \\
8.5008 \\
11.9995\end{array}$ & $\begin{array}{l}0.0165 \\
0.0181 \\
0.0075\end{array}$ \\
\hline NIT & & 4.8 & & 4.3 & & 4.4 & \\
\hline
\end{tabular}

IEE PROCEEDINGS-F, Vol. 139, No. 4, AUGUST 1992 
tions. We observed that the monopulse estimator was able to track the target with high accuracy during the entire period.

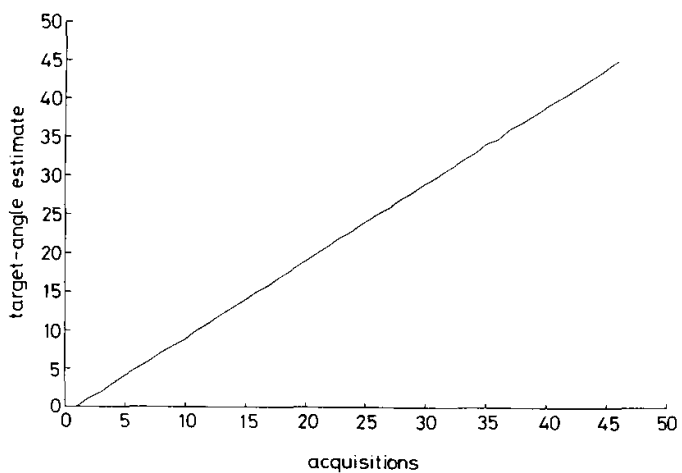

Fig. 2 Trajectory of target-angle estimates (in degrees) obtained by a 33-element LES monopulse array employing Chebyshev weighting with $-25 \mathrm{~dB}$ sidelobes. The target angle varied from $0^{\circ}$ to $45^{\circ}$. The SNR was $10 \mathrm{~dB}$. For each acquisition, the proposed $r t-M N_{p^{-2}}$ algorithm was executed for three iterations to obtain the angle estimate.

\section{Conclusion}

A novel fast iterative implementation of beamspace rootform eigen-based DOA estimators has been proposed. The new method avoids rooting a large-order polynomial, as is often required by conventional root-form methods. By judiciously constructing an augmented noise EV matrix, and converting it into banded form, the new method needs to work only with a polynomial of order $D$ at each iteration. The reduction in computation makes the new method suitable for real-time applications. Simu- lations show that, with appropriate preliminary DOA estimates, the new method converges in a few iterations and performs comparably to beamspace root-MUSIC.

\section{Acknowledgment}

This work was supported in part by the National Science Council of the ROC under contract NSC80-0404-E00905

\section{References}

BARABELL, A.J.: 'Improving the resolution performance of eigenstructure-based direction-finding algorithms'. Proc. ICASSP '83, 1983, pp. 336-339

2 KUMARESAN, R,, and TUFTS, D.W.: 'Estimating the angles of arrival of multiple plane waves', IEEE Trans., 1983, AES-19, pp. $134-138$

3 RAO, B., and HARI, K.: 'Performance analysis of root-MUSIC', IEEE Trans., 1989, ASSP-37, pp. 1939-1949

4 LI, F and VACCARO, R J. "Analytical performance prediction of subspace-based algorithms for DOA estimation'. SVD and Signal Processing II, Algorithms, Analysis and Applications (Elsevier, New York, 1991)

5 XU, X.L., and BUCKLEY, K.M.: 'Reduced-dimension beamspace broad-band localization: preprocessor design and evaluation'. Proc IEEE Fourth Workshop on Spectrum Estimation and Modeling 1988 , pp. 22-26

6 LEE H.B, and WENGROVITZ, M.S.: 'Resolution threshold of beamspace MUSIC for two closely spaced emitters', IEEE Trans. 1990, ASSP-38, pp. 1545-1559

7 ZOLTOWSKI, M.D., and LEE, T.S.: 'Maximum-likelihood based sensor array signal processing in the beamspace domain for lowangle radar tracking', IEEE Trans., 1991, SP-39, pp. 656-671

8 ZOLTOWSKI, M.D., KAUTZ, G., and SILVSERSTEIN, S. 'Development, performance analysis, and experimental evaluation of beamspace root-MUSIC'. Proc. ICASSP '91, 1991, pp. 3049-3052

9 SKOLNIK, M.I: 'Introduction to radar systems' (McGraw-Hill, New York, 1980)

10 STOICA, P., and SHARMAN, K.: 'Maximum-likelihood methods for direction-of-arrival estimation', IEEE Trans., 1990, ASSP-38, pp. $1132-1143$ 